\title{
Rate Theory for Correlated Processes: Double Jumps in Adatom Diffusion
}

Jacobsen, J.; Jacobsen, Karsten Wedel; Sethna, J.

Published in:

Physical Review Letters

Link to article, DOI:

10.1103/PhysRevLett.79.2843

Publication date:

1997

Document Version

Publisher's PDF, also known as Version of record

Link back to DTU Orbit

Citation (APA):

Jacobsen, J., Jacobsen, K. W., \& Sethna, J. (1997). Rate Theory for Correlated Processes: Double Jumps in Adatom Diffusion. Physical Review Letters, 79(15), 2843-2846. https://doi.org/10.1103/PhysRevLett.79.2843

\section{General rights}

Copyright and moral rights for the publications made accessible in the public portal are retained by the authors and/or other copyright owners and it is a condition of accessing publications that users recognise and abide by the legal requirements associated with these rights.

- Users may download and print one copy of any publication from the public portal for the purpose of private study or research.

- You may not further distribute the material or use it for any profit-making activity or commercial gain

- You may freely distribute the URL identifying the publication in the public portal

If you believe that this document breaches copyright please contact us providing details, and we will remove access to the work immediately and investigate your claim. 


\title{
Rate Theory for Correlated Processes: Double Jumps in Adatom Diffusion
}

\author{
Joachim Jacobsen, ${ }^{1}$ Karsten W. Jacobsen, ${ }^{1,2}$ and James P. Sethna ${ }^{1}$ \\ ${ }^{1}$ Laboratory of Atomic and Solid State Physics, Cornell University, Ithaca, New York 14853-2501 \\ ${ }^{2}$ Center for Atomic-scale Materials Physics, Department of Physics, Technical University of Denmark, DK-2800 Lyngby, Denmark
}

(Received 18 June 1997)

\begin{abstract}
We study the rate of activated motion over multiple barriers, in particular the correlated double jump of an adatom diffusing on a missing-row reconstructed platinum (110) surface. We develop a transition path theory, showing that the activation energy is given by the minimum-energy trajectory which succeeds in the double jump. We explicitly calculate this trajectory within an effective-medium molecular dynamics simulation. A cusp in the acceptance region leads to a $\sqrt{T}$ prefactor for the activated rate of double jumps. Theory and numerical results agree. [S0031-9007(97)04186-0]
\end{abstract}

PACS numbers: 68.35.Fx, 05.20.Dd, 82.20.Db

Reaction rates and diffusion rates in crystalline environments typically have an Arrhenius dependence on temperature $r_{1}=\nu \exp \left(-E_{\mathrm{TS}} / T\right)$. This asymptotic rate at low temperatures may be calculated using transition state theory (TST) [1], where $E_{\mathrm{TS}}$ is the energy of the saddlepoint atomic configuration separating the initial and final states (the transition state), and $\nu$ is a temperatureindependent prefactor involving the curvatures of the energy surface.

Many rates and transitions are not described by a simple crossing of a single barrier, and we should expect that their rates will not be given by the simple Arrhenius form. In this paper we study a double jump: a correlated diffusion event where an atom crosses two barriers. The development of field-ion microscopy and scanning tunneling microscopy has made it possible to track the motion of individual atoms at surfaces [2], and to directly measure the rates of these correlated transitions $[3,4]$. Developing what we call transition path theory (TPT), we show the rate is determined by the energy $E_{\mathrm{TP}}$ of the transition path (minimizing the energy among all paths which succeed in the double jump), and has the asymptotic form $r_{2}=C \sqrt{T} \exp \left(-E_{\mathrm{TP}} / T\right)$. We develop an efficient numerical method to calculate this minimumenergy path and the rate, and use it to describe double jumps along the troughs of the missing-row reconstructed $\mathrm{Pt}(110)$ surface within effective-medium theory [5] making contact with the Arrhenius double-jump rate measured in the recent Århus experiment [4].

Previously, the rates of double jumps have been discussed theoretically as so-called dynamical corrections to transition state theory [6,7] or in the context of generalized Langevin equation models $[8,9]$.

Consider an extra adatom on the reconstructed (110) surface (Fig. 1). A single-jump diffusion event is a thermal fluctuation in which the adatom moves along the troughs in the surface from one potential well over the transition barrier to a neighboring well (say from well A to $B$ in the right-hand side of Fig. 1). In a double jump, the atom moves from one potential well over two barriers before settling down in a well (A to $\mathrm{C}$ in Fig. 1). The double jumps can be distinguished from two subsequent single jumps only if there is a separation of time scales: the duration time for a double jump to pass through the central well (roughly a picosecond for Pt) has to be much smaller than the inverse rate $1 / r_{1}$ for single jumps (1.4 nsec at the highest temperatures we work at).

In the case of a single jump the transition state energy $E_{\mathrm{TS}}$ is the smallest possible thermal fluctuation for a single jump to occur. For our calculation, $E_{\mathrm{TS}}$ is $469.5 \mathrm{meV}$. What is the smallest possible thermal fluctuation in which the adatom performs a double jump, fluctuating to the top of one hill, sliding down, up to the top of a second hill, and
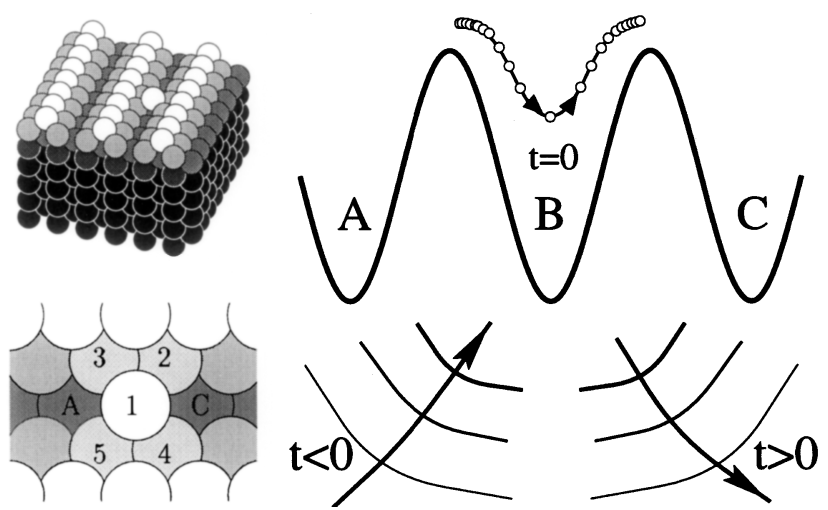

FIG. 1. The geometry of the $\operatorname{Pt}(110)$ surface with an adatom and the transition path (TP) for a double jump. At upper left, one sees the actual volume of atoms used in the simulations, with periodic boundary conditions and static atoms at the bottom. Lower left shows the geometry of the system with the adatom No. 1 in a valley formed by the reconstruction. At right we show schematically the TP and the energy. The TP starts at $t=-\infty$ with the system locally (close to the adatom) at the transition state (TS) potential energy saddle point between wells $\mathrm{A}$ and $\mathrm{B}$; in addition, there is an energy $\Delta E=E_{\mathrm{TP}}-E_{\mathrm{TS}}$ stored in the degrees of freedom far away. The additional energy radiates in from infinity and helps the adatom cross the well and get to the second TS. Our calculation shows that the adatom rolls off the hill, hits atoms 2 and 4 and knocks them aside, then is hit from behind by atoms 3 and 5, who boost the adatom up the other hill. As $t \rightarrow \infty$ the energy $\Delta E$ is again radiated away to infinity [10]. 
then down? Here the energy of the minimal fluctuation involves both kinetic and potential energy: we must look in phase space for the transition path: the lowest energy trajectory which will give us a double jump.

Before we more formally explain how the TP is calculated we shall describe the qualitative features of the path. Consider first the time evolution of the system if it is started out in a TS and the adatom is given a slight push. The adatom will then slowly leave the saddle point and slide down the hill into a well but it will not make it over the next barrier because energy will be transferred to other atoms in the system. Eventually the adatom will be at rest at the bottom of the well and all the energy $E_{\mathrm{TS}}$ will be in the degrees of freedom far away from the adatom. The TP is defined so that it brings the adatom to the top of the next barrier (the next TS) with the minimum additional energy $\left(\Delta E=E_{\mathrm{TP}}-E_{\mathrm{TS}}\right)$ necessary. In the TP the system is at $t=-\infty$ at the TS for all the degrees of freedom close to the adatom, but an energy $\Delta E$ is stored in the degrees of freedom far away. As the adatom slides down the hill the energy is radiating in from infinity helping the adatom up to the second TS (Fig. 1). As $t \rightarrow \infty$ the adatom (and all local coordinates/momenta) approaches the second TS and the energy $\Delta E$ is again radiated away to infinity [10]. The additional energy $\Delta E$ results in a higher activation energy for double jumps than for single jumps.

In finding the minimum energy double-jump path, it is important to use as variables the positions and momenta $\Gamma$ at $t=0$ when the adatom is crossing the bottom of the central well, because at this time the additional energy $\Delta E$ is most localized near the adatom. Let $p_{x}$ be the $x$ momentum of the adatom at $t=0$, where $x$ is the coordinate along the trough. To find $\Gamma_{\mathrm{TP}}$ of the TP, we specify the positions and the momenta of the nearby atoms within a radius $R$ of the adatom, and place atoms outside $R$ at their relaxed positions. We then vary $p_{x}$ to find $p_{x}^{\min }$, where the adatom just barely succeeds in the double jump: crosses into the well on the right at large times, and into the well on the left at large negative times [11]. We calculate the total energy, and minimize with respect to the positions and momenta of the nearby atoms. With all atoms in their relaxed positions, $652.0 \mathrm{meV}$ of energy is needed in $p_{x}$ to get a double jump. Optimizing degrees of freedom within $R=1,2.2,3.1,3.8$, and 4.9 nearest neighbor distances of the adatom, the total energy decreases to $621.7,595.8,591.4,590.2$, and $589.8 \mathrm{meV}$, respectively. The energy converges rapidly as more degrees of freedom are optimized and $\Gamma \rightarrow \Gamma_{\mathrm{TP}}$. We estimate $E_{\mathrm{TP}}$ to be $589.8 \mathrm{meV}: 120.3 \mathrm{meV}$ higher than the single jump $E_{\mathrm{TS}}$. The calculated TP appears to have both of the obvious possible symmetries: one reflection plane, and one reflection plane plus time reversal. The time reversal symmetry implies that the local atoms both start and end at zero velocity at the saddle points.

To calculate the rate at low temperatures, we need to sum over all the low-energy double-jump trajectories. Figure 2 gives an idea about how the double-jump region

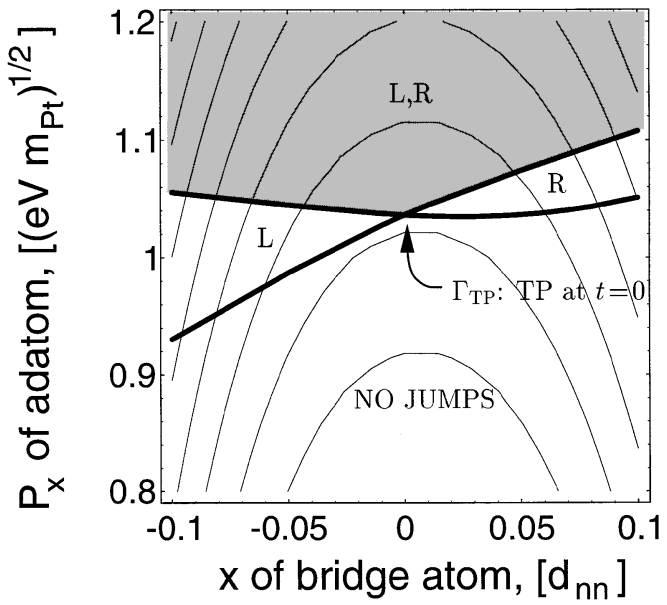

FIG. 2. Energy contours $(100 \mathrm{meV})$ in a cut in phase space. $m_{\mathrm{pt}}$ and $d_{\mathrm{nn}}$ are the atomic mass and nearest neighbor distance of platinum. The bridge atom is atom 2 in Fig. 1. All other coordinates are fixed at their values in the transition path (TP) at time $t=0$. L ( $\mathrm{R})$ indicates regions in phase space, where the adatom makes a jump to the left (right), and the solid lines are trajectories where the local atoms end at the saddle points. The double-jump region (gray shaded) is above both lines, and so the bounding surface for the double-jump region has a cusp passing through $\Gamma_{\mathrm{TP}}$, the phase-space point taken by the TP at $t=0$.

in phase space looks. The figure shows this region in a two-dimensional cut in phase space (the plane spanned by the $x$ coordinate of a bridge atom (atom 2 in Fig. 1) and $p_{x}$ of the adatom; all the remaining coordinates are at their $\Gamma_{\mathrm{TP}}$ values). $\mathbf{R}$ is the region corresponding to trajectories where the adatom will jump over the barrier to the right; $\mathbf{L}$ is the region where the adatom came over the barrier from the left. The TP is the lowest-energy trajectory which came from the left and makes it to the right, and is situated on the cusp at the (codimension two) intersection of the bounding surfaces of $\mathbf{R}$ and $\mathbf{L}$.

Our method for calculating the rate of double jumps is analogous to the standard TST method for calculating the rates for single jumps. TST expresses the hopping rate as the flux through the dividing surface; at low temperatures it is well described as a harmonic expansion of the energy about the TS [1]:

$$
\begin{aligned}
r_{1}^{\mathrm{TST}} & =\left\langle\Theta\left(v_{r}\right) v_{r} \delta\left(x_{r}-x_{r}^{b}\right)\right\rangle / Z_{W} \\
& =\frac{1}{2 \pi} \frac{\prod_{i=1}^{3 N} \omega_{i}}{\prod_{i=1}^{3 N-1} \omega_{i}^{\mathrm{TS}}} \exp \left(-E_{\mathrm{TS}} / T\right) .
\end{aligned}
$$

In this expression $\langle\ldots\rangle$ denotes a thermal average, and $Z_{W}$ denotes the partition function in one well. The reaction coordinate $x_{r}$ equals $x_{r}^{b}$ at the top of the barrier, the $\omega$ 's denote the eigenfrequencies at the bottom of the well, and the $\omega^{\mathrm{TS}}$, s denote the eigenfrequencies found by the harmonic expansion at the TS (excluding the imaginary, unstable frequency of the saddle point). Choosing the coordinate $x_{r}$ to be along the eigendirection of the imaginary frequency minimizes the recrossing corrections [1]. 
Similarly, the double-jump rate can be calculated as the thermal average of the flux through a surface $\tilde{x}=0$ due to double-jump trajectories:

$$
r_{2}=\left\langle\Theta_{d j} v_{\tilde{x}} \delta(\tilde{x})\right\rangle / Z_{W} .
$$

Here $\Theta_{d j}$ is one if the trajectory is a double jump, and zero otherwise. In both cases the rate is calculated as a flux through a surface, with a $\Theta$ function keeping only trajectories which succeed in the transition.

We generate an importance-sampling distribution of $M$ trajectories, $\left\{\Gamma_{\alpha}\right\}$, in the neighborhood of the transition path by adding $\Gamma_{\mathrm{TP}}$ to a thermal ensemble restricted to $\tilde{x}=0$ at the bottom of the well. We found it important numerically to choose $\tilde{x}$ to be a relative coordinate: the difference between the $x$ coordinate of the adatom and the average $x$ coordinate of the four bridge atoms (Nos. 2-5 in Fig. 1). We then calculate the ratio of the double-jump to single-jump rates:

$$
\begin{aligned}
\frac{r_{2}}{r_{1}}= & \frac{\left\langle\Theta_{d j} v_{\tilde{x}} \delta(\tilde{x})\right\rangle}{\left\langle\Theta\left(v_{r}\right) v_{r} \delta\left(x_{r}-x_{r}^{b}\right)\right\rangle} \\
= & \frac{\langle\delta(\tilde{x})\rangle\left\langle\Theta_{d j} v_{\tilde{x}} \delta(\tilde{x})\right\rangle}{\left\langle\Theta\left(v_{r}\right) v_{r}\right\rangle\left\langle\delta\left(x_{r}-x_{r}^{b}\right)\right\rangle\langle\delta(\tilde{x})\rangle} \\
= & \sqrt{2 \pi m / T}\left[e^{E_{\mathrm{TS}} / T} \prod_{i=1}^{3 N-1} \frac{\omega_{i}^{\mathrm{TS}}}{\tilde{\omega}_{i}}\right] \\
& \times \frac{1}{M} \sum_{\alpha=1}^{M} v_{\tilde{x}, \alpha} \Theta_{d j}\left(\Gamma_{\alpha}\right) e^{\left[E\left(\Gamma_{\alpha}-\Gamma_{\mathrm{TP}}\right)-E\left(\Gamma_{\alpha}\right)\right] / T} .
\end{aligned}
$$

We determine which trajectories are double jumps numerically: we run the molecular dynamics trajectory forward and backward in time for a few picoseconds until the trajectory either recrosses the original well (failure, $\Theta_{d j}=0$ ) or crosses the bottom of both of the adjacent wells (success, $\Theta_{d j}=1$ ). This expression is complicated by the fact that we are measuring the flux for single jumps at the top of the barrier, and the flux for double jumps at the bottom of the well: the term in square brackets is precisely the ratio of probabilities of being at these two planes, in the harmonic approximation (the $\tilde{\omega}$ 's being the eigenfrequencies in the plane $\tilde{x}=0$, and we evaluate $\prod \omega_{i}^{\mathrm{TS}} / \tilde{\omega}_{i}$ to be 1.722 ). Our double-jump term is fully nonlinear.

Figure 3 shows the "TP MD" (transition path molecular dynamics) values calculated using Eq. (3). Also shown are rate calculations performed using the more traditional method described by Voter and Doll [6] (transition state molecular dynamics "TS MD"), where trajectories are started with a thermal distribution at the TST dividing surface, and the fraction of double jumps is directly measured. Within the statistical error bars the two methods give the same rate, as they should. However, the uncertainty in the traditional rate determination increases drastically at lower temperatures because the fraction of double-jump trajectories in the thermal ensemble becomes small. In our language, the old method centers attention not at $\Gamma_{\mathrm{TP}}$, but far away at the single-jump transition state.

We now discuss the asymptotic behavior of the doublejump rates at low temperatures. In analogy to the

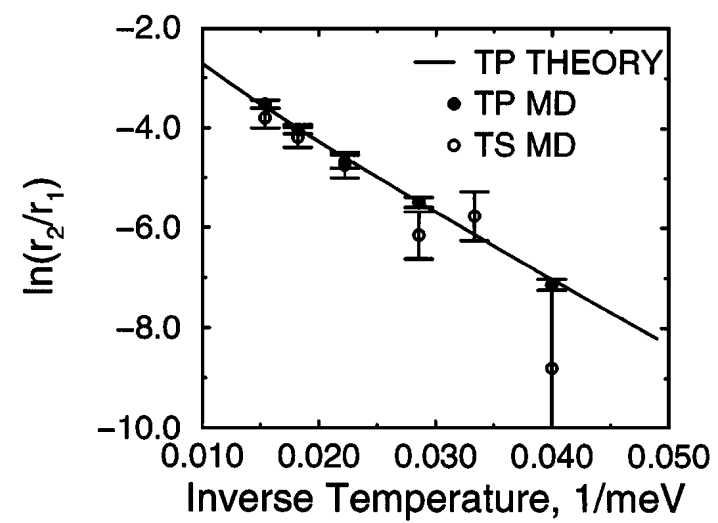

FIG. 3. Arrhenius plot of the rate of double jumps relative to single jumps, showing the transition state molecular dynamics (TS MD), transition path molecular dynamics (TP MD), and transition path theory results. The TP theory curve is $r_{2} / r_{1}=$ $C_{2} \sqrt{T} \exp -\left(E_{\mathrm{TP}}-E_{\mathrm{TS}}\right) / k T$, where the one parameter $C_{2}$ is fitted to the TP MD data to be $0.022 \mathrm{meV}^{-1 / 2}$.

harmonic expansion around the TS which leads to the TST rate with an Arrhenius behavior for the single-jump rate [Eq. (1)] we can define a transition path theory rate $r_{2}^{\mathrm{TPT}}$ for the double jumps by performing an expansion of Eq. (2) around the TP.

$\Theta_{d j}$ is a Heaviside step function in $p_{x}-p_{x}^{\min }(\tilde{\Gamma})$, (Fig. 2), where $\tilde{\Gamma}$ is the phase-space point at $t=0$ excluding $x$ and $p_{x}$ of the adatom. The $x$ and $p_{x}$ parts of the phase-space integral in the numerator of Eq. (2) (choosing here for convenience $\tilde{x}=x$ ) can be explicitly carried out, leading to

$$
r_{2}=\frac{T}{Z_{W}} \int d \tilde{\Gamma} \exp [-\tilde{E}(\tilde{\Gamma}) / T],
$$

where $\tilde{E}(\tilde{\Gamma})=E\left(x=0, p_{x}^{\min }(\tilde{\Gamma}), \tilde{\Gamma}\right)$ is the energy on the boundary of the double-jump region. In the lowtemperature limit a harmonic expansion of $\tilde{E}(\tilde{\Gamma})$ around the TP in the $6 N-2$ variables $\tilde{\Gamma}$ can be performed and the resulting multidimensional Gaussian integral can in principle be carried out. Focusing on the temperature dependence of the prefactor, we note that $p_{x}^{\min }$, and hence $\tilde{E}$ has a cusp in one degree of freedom (see Fig. 2), and that there is a $(6 N-3)$-dimensional subspace, where the energy is presumably locally quadratic. These $6 N-3$ degrees of freedom should give rise to a factor $\sqrt{T}$ (each) in the prefactor, whereas the cusp degree of freedom gives by integration a factor $T$, so overall we get the prefactor $T \times T^{(6 N-3) / 2} \times T / T^{3 N}=\sqrt{T}$. The TPT rate for the double jumps is therefore of the form

$$
r_{2}^{\mathrm{TPT}}=C_{1} \sqrt{T} \exp \left(-E_{\mathrm{TP}} / T\right),
$$

with $C_{1}$ a temperature-independent constant. Alternatively we may write $r_{2}^{\mathrm{TPT}} / r_{1}^{\mathrm{TST}}=C_{2} \sqrt{T} \exp \left[-\left(E_{\mathrm{TP}}-\right.\right.$ $\left.\left.E_{\mathrm{TS}}\right) / T\right]$, where $C_{2}$ is the constant $C_{1} / \nu$.

The TPT-rate expression is shown as the solid curve in Fig. 3 with only the constant $C_{2}$ fitted to the calculated rates. The TPT rate is clearly in agreement with the 


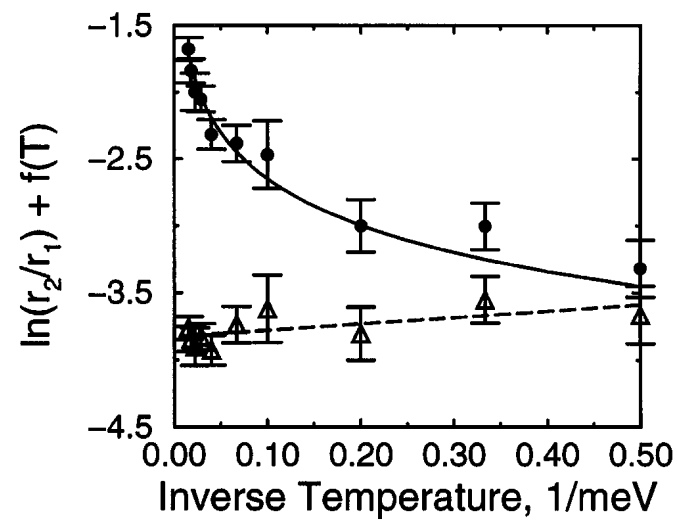

FIG. 4. Temperature dependence of TP MD data for the rate of double jumps relative to single jumps. The black dots show the data corrected for the predicted exponential temperature dependence $\left[f(T)=\Delta E / T, \Delta E=E_{\mathrm{TP}}-E_{\mathrm{TS}}=\right.$ $120.3 \mathrm{meV}]$. The curvature of this set of points proves that the prefactor depends on temperature, and is well described by the solid line, which is a plot of $\ln C_{2} \sqrt{T}$, where $C_{2}$ is $0.022 \mathrm{meV}^{-1 / 2}$ as in Fig. 3. The open triangles show the data now also corrected for the $\sqrt{T}$ dependence $[f(T)=$ $\Delta E / T-\ln T / 2]$. TP theory predicts this set of points is to be a constant $\left(\ln C_{2}\right)$, consistent with the data. Linear regression (dashed line) gives a slope of $+0.47 \pm 0.2 \mathrm{MeV} \sim 0.4 \%$ of $\Delta E$. This slope is a reasonable estimate of how much further our estimated $E_{\mathrm{TP}}$ could be lowered by further optimization.

simulation results even up to the highest temperature $T=65 \mathrm{meV}$. At high temperatures corrections caused by anharmonic effects can be expected and higher order multiple jumps (triple, quadruple, etc.) may also play a role in the diffusivity [12]. It is easy to fit the TP MD data of Fig. 3 with an Arrhenius form with constant prefactor, but it yields the wrong energy barrier $(139 \mathrm{meV}, 16 \%$ too high).

Since we sample the vicinity of the TP in our numerical method, we can extend the calculation of the double-jump rate to much lower temperatures to confirm the theoretical asymptotic behavior. (The double-jump rate becomes very small.) In Fig. 4 we show the result for $r_{2} / r_{1}$ dividing out first the predicted Arrhenius dependence (solid symbols), and further the predicted $\sqrt{T}$ dependence (open symbols), ending up with a set of data points consistent with a constant. Hence our numerical method confirms the TPT rate as given by Eq. (5).

Comparing with the recent experiments [4] on the $\mathrm{Pt} / \mathrm{Pt}(110)$ system we note that in our model the barrier for single jumps is $E_{\mathrm{TS}}=469.5 \mathrm{meV}$ while the activation energy experimentally is found to be around $0.8 \mathrm{eV}$. It is well known that the effective medium theory potential tends to underestimate diffusion barriers for Pt [13]. However, the TPT analysis confirms the experimental observation of a thermally activated form; the calculated additional activation energy for double jumps $E_{\mathrm{TP}}-E_{\mathrm{TS}}=$ $120 \mathrm{meV}$ is in perhaps accidentally good agreement with the experimentally determined value which is of the order $0.1 \mathrm{eV}$.
We finally note that we have applied the TPT to the traditional Langevin equation (Ohmic damping) previously used to describe double jumps [8]. Here the TP becomes the optimal time evolution of the external "fluctuating" force. The additional activation energy $\Delta E$ for double jumps equals the work done by friction in the adiabatic potential only in the low friction limit. We observe the same $\sqrt{T}$ prefactor [8].

The authors thank Flemming Besenbacher, Trolle Linderoth, Christopher Henley, and Per Stoltze for helpful conversations. This work was supported by the National Science Foundation through the Cornell Materials Science Center NSF-DMR-9121654. Support was also provided by the Department of Energy DEFG0288-ER45364, Cornell Theory Center, and the Danish National Research Foundation through the Center for Atomic-scale Materials Physics.

[1] P. Hänggi, P. Talkner, and M. Borkovec, Rev. Mod. Phys. 62, 251 (1990).

[2] G. Ehrlich and F. G. Hudda, J. Chem. Phys. 44, 1039 (1966); T. T. Tsong, Phys. Rev. B 6, 417 (1972); G. Ehrlich, Surf. Sci. 246, 1 (1991); G. Kellogg, Surf. Sci. Rep. 21, 1 (1994); B. S. Schwartzentruber, Phys. Rev. Lett. 76, 459 (1996).

[3] D. C. Senft and G. Ehrlich, Phys. Rev. Lett. 74, 294 (1995).

[4] T. R. Linderoth, S. Horch, E. Lægsgaard, I. Stensgaard, and F. Besenbacher, Phys. Rev. Lett. 78, 4978 (1997).

[5] K. W. Jacobsen, J. K. Nørskov, and M.J. Puska, Phys. Rev. B 35, 7423 (1987); K. W. Jacobsen, Comments Condens. Matter Phys. 14, 129 (1988); K. W. Jacobsen, P. Stoltze, and J. K. Nørskov, Surf. Sci. 366, 394 (1996).

[6] A. F. Voter and J. D. Doll, J. Chem. Phys. 82, 80 (1985).

[7] For example, Z. Zhang, K. Haug, and H. Metiu, J. Chem. Phys. 93, 3614 (1990); K. D. Dobbs and D. J. Doren, J. Chem. Phys. 97, 3722 (1992).

[8] Y. Georgievskii, M. A. Kozhushner, and E. Pollak, J. Chem. Phys. 102, 6908 (1995); Y. Georgievskii and E. Pollak, Surf. Sci. Lett. 355, L366 (1996); E. Pollak (private communication).

[9] R. Ferrando, R. Spadacini, G. E. Tommei, and G. Caratti, Surf. Sci. 311, 411 (1994).

[10] In our system, a small amount of energy is left in a longlived local mode with the adatom oscillating vertically about the TS; this energy will also eventually decay due to anharmonicity.

[11] An efficient way to determine $p_{x}^{\min }$ in practice is by maximizing the time $t$ it takes for the adatom to get to the dividing surface, using that $\left|p_{x}-p_{x}^{\min }\right| \propto \exp \left(-4 \pi \nu_{\mathrm{TS}} t\right)$ for large $t$, where $\nu_{\mathrm{TS}}$ is the frequency of the unstable mode at the saddle point.

[12] We note that we in principle could determine the TP's and the corresponding TPT rates also for triple or longer jumps.

[13] J. Jacobsen, K. W. Jacobsen, P. Stoltze, and J. K. Nørskov, Phys. Rev. Lett. 74, 2295 (1995). 